\title{
Expression of Helicobacter pylori ggt Gene in Baculovirus Expression System and Activity Analysis of its Products
}

\author{
MEI KONG, MING XU, YA-LONG HE and YOU-LI ZHANG* \\ Department of Gastroenterology, the Affiliated Hospital of Jiangsu University
}

Received 21 December 2010, revised 26 June 2011, accepted 6 July 2011

Abstract

\begin{abstract}
The $\gamma$-glutamyltranspeptidase (GGT) of Helicobacter pylori (HpGT) is a newly found virulence factor. In an approach to gain insight into the gene function, the four domains of the HpGT were cloned and expressed in baculovirus expression system. The results of a functional assay showed that the HpGT products acted as GGT, even when the N-terminal 380 amino acids were deleted. However, only the full length open reading frame (ORF) of the HpGT gene was apparently effective on cell growth. This result indicated that the products of the full length ORF might have an important role in gastric carcinogenesis. In this paper, we are the first to report that changes of mitochondrial membrane potential can be detected using 5, 5', 6, 6'-tetrachloro-1, 1', 3, 3'-tetraethylbenzimidazole carbocyanine iodide (JC-1) staining in insect cells.
\end{abstract}

Ke y words: glutamyltranspeptidase; Helicobacter pylori; membrane potential; virulence factor

\section{Introduction}

Helicobacter pylori is a common human pathogen that colonizes the gastric mucosa. Infection puts the individual at greater risk for developing gastritis, peptic ulcer disease, and gastric cancer (Marshall and Warren, 1984; Bjorkholm et al., 2003; Sharma and Vakil, 2003). $H$. pylori produces a number of virulence factors that enable its pathogenesis. One of these is an enzyme, $\gamma$-glutamyltranspeptidase (GGT, EC 2.3.2.2), in the periplasm, which is involved in induction of host cell apoptosis (Shibayama et al., 2003) and plays an important role in colonization by H. pylori (Chevalier et al., 1999; McGovern et al., 2001).

GGTs are fairly ubiquitous with homologues observed in all kingdoms, and are generally considered to be involved in the metabolism of glutathione and in the salvaging of cysteine (Hanigan and Ricketts, 1993; Ikeda and Taniguchi, 2005). Although distant GGTs often share considerable sequence identity, significant catalytic differences exist between bacterial and nonbacterial homologues (Ikeda et al., 1995; Ikeda et al., 1996). Mammalian GGTs are embedded in the plasma membrane by a single $\mathrm{N}$-terminal transmembrane anchor and are heterologously glycosylated, bacterial homologs are soluble and localized to the periplasmic space. H. pylori GGT (HpGT) is a member of the $\mathrm{N}$-terminal nucleophile hydrolase superfamily, and is translated as an inactive proenzyme that undergoes autoprocessing to become an active enzyme (Brannigan et al., 1995). Despite its demonstrated involvement in $H$. pylori colonization, persistence, and disease progression (Boanca et al., 2007), the biochemical characterization of HpGT is still limited (Coloma and Pitot 1986). Although the general features of the function of $\mathrm{HpGT}$ can be inferred based on its classification as an N-terminal nucleophile hydrolase, many mechanistic details of the autoactivation and catalytic function of HpGT have not been addressed. In this paper, the domains of the HpGT gene were expressed in baculovirus expression system and subsequently analyzed.

\section{Experimental}

\section{Materials and Methods}

The E. coli DH10Bac/BmNPV cell line was provided by Professor E.Y. Park (Department of Applied Biological Chemistry, Faculty of Agriculture, Shizuoka University, Shizuoka, Japan).

FuGENE $^{\mathrm{TM}} 6$ transfection reagent was from Roche Company. Grace's insect cell culture medium (GIBCO) was purchased from Invitrogen. The B. mori cell line $\mathrm{BmN}$ (originated from ovary) was preserved in our laboratory and cultured at $27^{\circ} \mathrm{C}$ in Grace's insect cell culture medium.

H. pylori culture. H. pylori strain was isolated from clinic tissues. The strains were grown on horse blood

\footnotetext{
* Corresponding author: Y-L. Zhang, Department of Gastroenterology, the Affiliated Hospital of Jiangsu University; Jiefang Road 438, Zhenjiang 212001, Jiangsu Province, China; e-mail: youlizhang1972@126.com
} 
Table I

The primers for amplification of HpGT fragments

\begin{tabular}{|l|lll|}
\hline Fragmnent & \multicolumn{2}{|c|}{ Primer } & Length (bp) \\
\hline Full length & F: cgc gaa ttc atg aga cgg agt ttt tta aaa acg $\quad$ (EcoR I) & 1704 \\
\hline $\mathrm{d} 30 \mathrm{a}$ & F: cgc gaa ttc atg ccc att aaa aac act aaa gtg $\quad($ Eco R I) & 1614 \\
\hline $\mathrm{d} 80 \mathrm{a}$ & F: cgc gaa ttc atg aat att ggt ggg ggg ggt ttt $\quad($ Eco R I) & 1464 \\
\hline $\mathrm{d} 380 \mathrm{a}$ & $\begin{array}{l}\text { F: cgc gaa ttc atg acg cat tat tct gta gcg ga } \\
\text { R: gac } \text { ctc gag aaa ttc ttt cct tgg atc cgt t }\end{array}$ & 564 \\
\hline eGFP & $\begin{array}{l}\text { F: gac ctc gag atg gtg agc aag ggc gag ga } \\
\text { R: cgc aag ctt tta ctt gta cag ctc gtc ca }\end{array}$ & (Xho I) \\
\hline
\end{tabular}

agar plates (Oxoid Base) supplemented with vancomycin $(5 \mathrm{mg} / \mathrm{L})$, polymyxin B $(2500 \mathrm{U} / \mathrm{L})$, trimethoprim $(5 \mathrm{mg} / \mathrm{L})$ and amphotericin B $(4 \mathrm{mg} / \mathrm{L})$. Plates were incubated in an anaerobic jar with a microaerobic gas in the presence of a catalyst. E. coli strain DH5a was used as hosts for plasmid cloning experiments, and was grown in L-broth ( $10 \mathrm{~g}$ of tryptone, $5 \mathrm{~g}$ of yeast extract and $5 \mathrm{~g}$ of $\mathrm{NaCl}$ per litre, $\mathrm{pH} 7.0$ ) or on L-agar plates (1.5\% agar) at $37^{\circ} \mathrm{C}$.

Cloning of the domains of HpGT gene. According to the HpGT gene structure and the DNA sequence in GenBank (Access No:NC_000921), four domains were designed for the functional analysis, including full length of the HpGT ORF (open reading frame), HpGTd30a, HpGT-d80a, and HpGT-d380a (deletion of 30, 80,380 amino acids in the $\mathrm{N}$-terminal, respectively). The specific primers for amplification of the four fragments are shown in Table I.

The PCR conditions for amplification of the HpGT fragments were: 1 cycle at $94^{\circ} \mathrm{C}$ for $5 \mathrm{~min} ; 35$ cycles at $94^{\circ} \mathrm{C}$ for $45 \mathrm{~s}, 55^{\circ} \mathrm{C}$ for $40 \mathrm{~s}, 72^{\circ} \mathrm{C}$ for $3 \mathrm{~min}$; and 1 cycle at $72^{\circ} \mathrm{C}$ for $10 \mathrm{~min}$.

Construction of recombinant donor plasmids. According to the HpGT protein structure, we designed four fragments (full length, d30a, d80a, and d380a) to analyze enzymic activity. After PCR, four fragments were obtained (Fig. 1a). Sequencing results indicated that no no-sense mutations occurred. The fragments were inserted into the baculovirus transfer vector pFastBac1. Furthermore, to detect the expression of the fragments, the fusion proteins with the eGFP gene were constructed in the C-terminal of the fragments.

The DNA of baculovirus donor plasmid pFastBac1 was digested with EcoR I and Xho I, and ligated to the HpGT fragments digested with the same enzymes, respectively. To generate fusing ORFs with eGFP gene, the eGFP fragment was inserted into the constructed plasmids containing the HpGT fragments by digestion with Xho I and Hind III as well.

Isolation of recombinant bacmid and baculoviruses. The recombinant bacmids were transformed into E.coli DH10Bac/BmNPV where transposition occurred. By screening the transformed clones, Bacmid
DNA was isolated using the FlexiPrep kit (Amersham Pharmacia Biotech) and then analyzed by PCR with the M13 primers (Su et al., 2009). The PCR conditions were 1 cycle at $94^{\circ} \mathrm{C}$ for $5 \mathrm{~min} ; 35$ cycles at $94^{\circ} \mathrm{C}$ for $45 \mathrm{~s}$, $55^{\circ} \mathrm{C}$ for $45 \mathrm{~s}$, and $72^{\circ} \mathrm{C}$ for $5 \mathrm{~min}$; and $1 \mathrm{cycle}$ at $72^{\circ} \mathrm{C}$ for $10 \mathrm{~min}$. Recombinant bacmid DNA confirmed by PCR was transfected into Bm cells using transfection reagent according to the manual.

$\gamma$-Glutamyltranspeptidase activity. After infection with the recombinant viruses p.i. $60 \mathrm{~h}$, the $\mathrm{BmN}$ cells were collected and lysed. The lysate with $100 \mu \mathrm{g}$ total protein was used for $\gamma$-Glutamyltranspeptidase activity assay. Qualitative detection of the GGT enzyme was achieved by a Clinic Biochemistry detector (Olympus AU2700), the tests were repeated 3 times.

Western-blot. To comfirm the protein expression, the products of fusing gene (the HpGT fragment with eGFP) were detected by Western-blot. The first antibody was of anti-eGFP with a dilution of 1:1000.

Analysis of mitochondrial membrane potential. After infected with the baculoviruses containing the HpGT fragment, respectively, mitochondrial membrane potential of the $\mathrm{BmN}$ cells was observed by fluorescence microscopy with JC-1 staining. The staining process was carried out by the protocol of the JC-1 Mitochondrial Membrane Potential Detection Kit (Biotium, Inc., Cat: 30001$)$. In brief, the living cells were stained red, and when the cells were going to die of apoptosis, the cells were stained green (Bowser et al., 1998).

MTT assay. The MTT assay was performed using the cell proliferation kit I MTT (Roche Co.) according to the manufacturer's protocol. In brief, after diverse treatments for $24 \mathrm{~h}$, the gastric cancer cells (BGC-823) were subsequently prepared for assays. The absorbance was determined at $570 \mathrm{~nm}$ by an ELISA reader (Bio-tech Synergy HT). Each approach was replicated 6 times and repeated 3 times with similar results.

\section{Results and discussion}

Identification and harvest of the recombinant bacmids. The HpGT fragments were amplified from Hp genomic DNA by PCR, and digested with EcoR I 


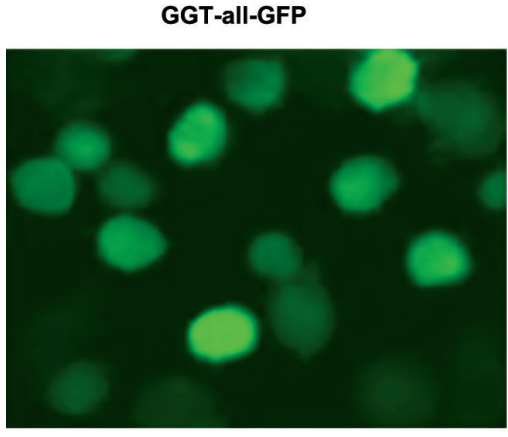

GGT-d80a-GFP

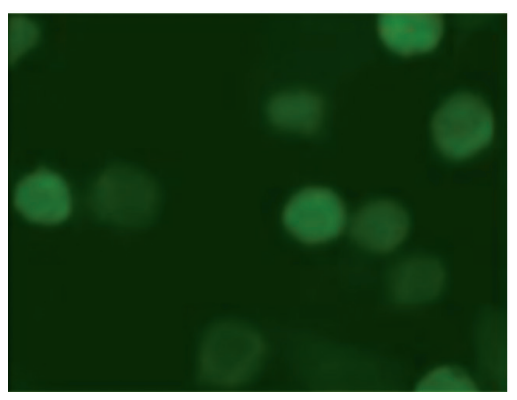

Fig. 1. Observation of HpGT products fusing with eGFP under fluorescent microscope (200-fold).

The GGT-d380a-eGFP products had different location in BmN cells. Bm-eGFP recombinant baculovirus, with egfp gene instead of the polyhedron gene, was a control.

Bm-GFP

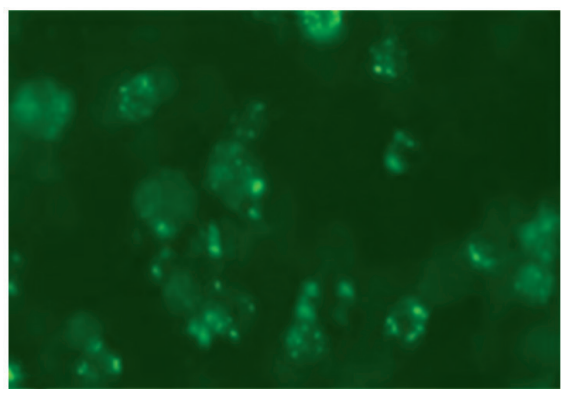

and Xho I to be inserted into the baculovirus transfer vector digested with the same enzymes. After the plasmids were transferred into the $\mathrm{Bm}-\mathrm{DH} 10$ bacteria, the bacmid DNAs were extracted, respectively. To confirm the fragment inserts, PCR was used to amplify the fragments. The results indicated that the HpGT fragments were inserted into the viral genome.

To construct the fusing fragments with eGFP, the eGFP fragments were amplified and were digested with Xho I and Hind III, and then ligated to the recombinant vectors containing the HpGT fragment.

A

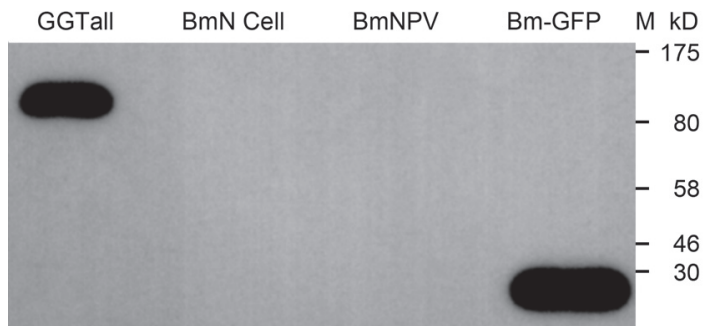

B
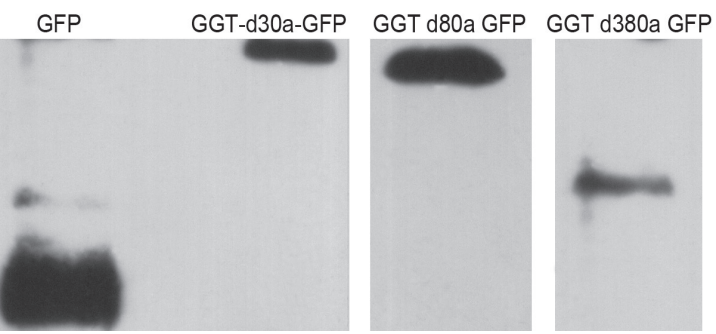

Fig. 2a and 2b. Detection of expression products of the fusing fragment of $g g t$ and $g f p$ in baculovirus by Western-blot.

The lysates of BmN cells and of cells infected with wild baculovirus (BmNPV) were the negative controls. In Figure 2a, the products of the full length $g g t$ fusing with eGFP gene were detected, and in Figure $2 b$, the products of the fragments of $g g t$ fusing with eGFP gene were detected.
The recombinant bacmid DNAs were transfected into $\mathrm{BmN}$ cells to generate budded viruses. After $72 \mathrm{~h}$ infection, the symptoms of viral infection were observed using an inverted phase microscope and the medium was collected as viral stock. Green fluorescence was observed in the infected cells when the HpGT fragments fusing eGFP were expressed (Fig. 1).

Confirmation of fusing expression. At of yet, the HpGT antibody has not been raised. Expression of the HpGT fragments was tested by tagging with eGFP gene. Using eGFP antibody, expression was confirmed by Western blot (Fig. 2a and 2b). After that, the location of the HpGT fragments in the cells was observed under an inverted fluorescent microscope. Interestingly, the fragments that lost $\mathrm{N}$-terminal 380aa were concentrated in the cytoplasm (Fig. 1).

GGT activity assay. The test of GGT activity assay showed that all the fragments used in this paper had GGT activity, even after deleting the N-terminal 380aa

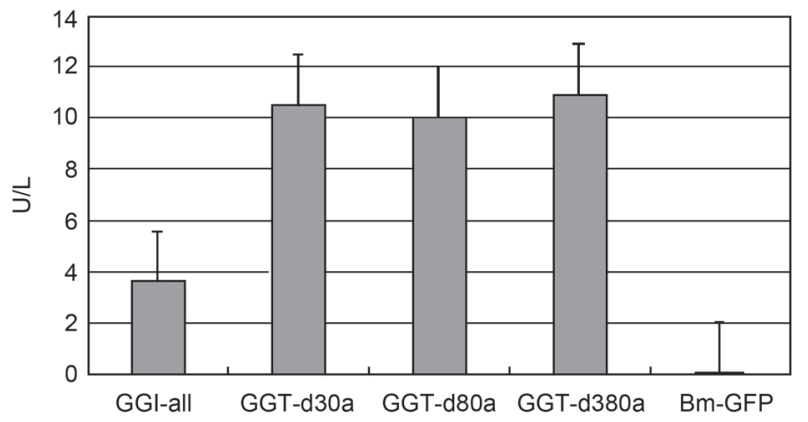

Fig. 3. GGT activity detection of the expression products of the fusing fragment of the ggt in baculovirus.

The Bm-GFP virus was a negative control. 

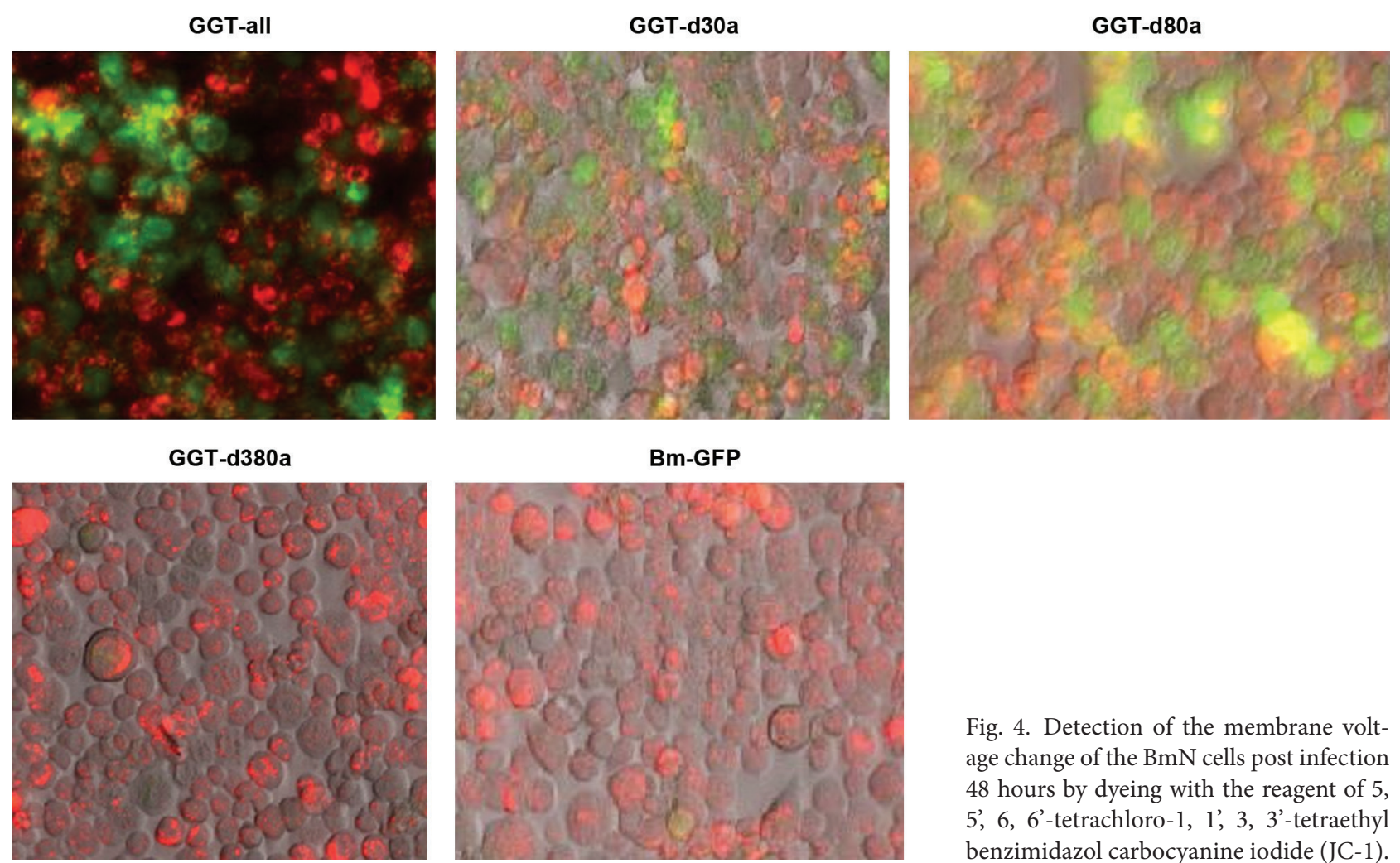

Fig. 4. Detection of the membrane voltage change of the $\mathrm{BmN}$ cells post infection 48 hours by dyeing with the reagent of 5 , 5', 6, 6'-tetrachloro-1, 1', 3, 3'-tetraethyl benzimidazol carbocyanine iodide (JC-1).

(Fig. 3). The result indicated that the enzyme activity center was located in the $\mathrm{C}$-terminal of the protein and the $\mathrm{N}$-terminal of HpGT might have other functions.

Mitochondrial membrane potential assay. To know the effect of the HpGT products on the cell cycle, the mitochondrial membrane potential of the infected $\mathrm{BmN}$ cells was compared. The results demonstrated that infection of HpGT fragments with full length, deleting $30 \mathrm{aa}$ and 80aa changed the mitochondrial membrane potential significantly, but the wild type virus and the HpGT fragment with deletion of 380aa did not change

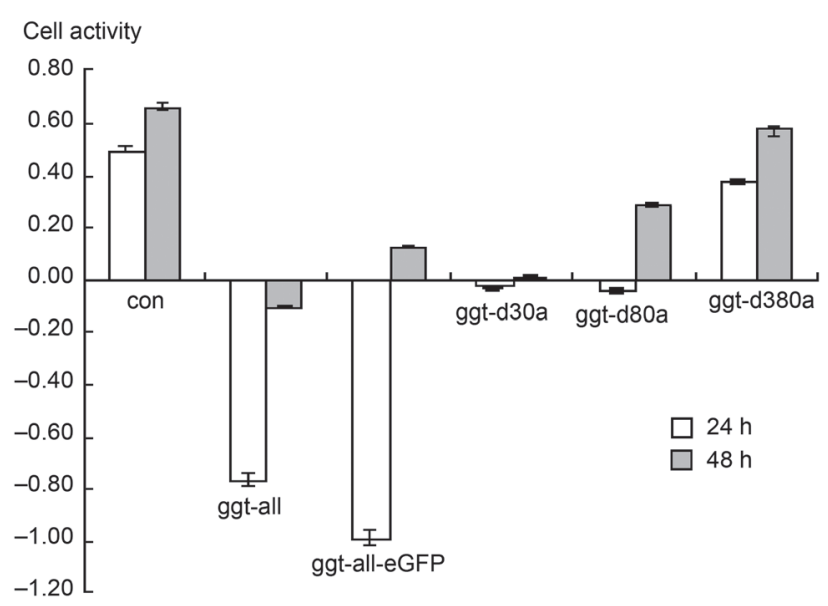

Fig. 5. Growth inhibition assay of BGC-823 cells (from gastric cancer) induced with the GGT expression products using MTT method. Only the products of the full length GGT and that fusing with GFP affected cell activity at 24 hours time point.Table I. The primers for amplification of HpGT fragments it apparently. This evidence denotes that the $\mathrm{N}$-terminal domain is related to cell function (Fig. 4). This is the first report using JC-1 staining to detect cell changes in insect $\mathrm{BmN}$ cells.

Effect of tumor cell growth. To check the impact of the HpGT products on cell survival, stomach cancer cells (BGC-823) were treated with the expression compound. Analysis of cell viability by MTT assay indicated that the cell growth was inhibited significantly by HpGT full length products at $24 \mathrm{~h}$. However, when the incubation time lasted $48 \mathrm{~h}$, cell growth showed recovery. These results indicate that only the full length HpGT might have the activity to inhibit cell growth (Fig. 5). Furthermore, the effect might be reversible.

In this paper, we identified the HpGT activity and effect on cell growth using baculovirus expression system. Although the C-terminal domain of the HpGT has GGT activity, only the full length ORF affected cell growth. This indicates that the products of the full length ORF may have an important role in gastric carcinogenesis.

\section{Acknowledgements}

This work was funded by the Science and Technology Development Foundation of Jiangsu University medicine and clinic (JLY2010106).

\section{Literature}

Bjorkholm B., P. Falk, L. Engstrand and O. Nyren. 2003. Helicobacter pylori: resurrection of the cancer link. J. Intern. Med. 253: 102-119. Boanca G., A. Sand, T. Okada, H. Suzuki, H. Kumagai, K. Fukuyama and J.J. Barycki. 2007. Autoprocessing of Helicobacter pylori 
$\gamma$-Glutamyltranspeptidase Leads to the Formation of a ThreonineThreonine Catalytic Dyad. J. Biol. Chem. 282: 534-541.

Bowser D.N., T. Minamikawa, P. Nagley and D.A. Williams. 1998. Role of mitochondria in calcium regulation of spontaneously contracting cardiac muscle cells. Biophys. J. 75: 2004-2014.

Brannigan J.A., G. Dodson, H.J. Duggleby, P.C. Moody, J.L. Smith, D.R. Tomchick and A.G. Murzin. 1995. A protein catalytic framework with an N-terminal nucleophile is capable of self-activation. Nature 378, 416-419.

Chevalier C., J.M. Thiberge, R.L. Ferrero and A. Labigne. 1999. Essential role of Helicobacter pylori gamma-glutamyltranspeptidase for the colonization of the gastric mucosa of mice. Mol. Microbiol. 31: 1359-1372.

Coloma J. and H.C., Pitot. 1986. Characterization and sequence of cDNA clone of gamma-glutamyltranspeptidase. Nucleic. Acids. Res. 14: 1393-1403.

Hanigan M.H. and W.A. Ricketts. 1993. Extracellular glutathione is a source of cysteine for cells that express gamma-glutamyl transpeptidase. Biochemistry 32: 6302-6306.

Ikeda Y., J. Fujii and N. Taniguchi. 1996. Effects of substitutions of the conserved histidine residues in human $\gamma$-glutamyl transpeptidase. J. Biochem. 119: 1166-1170.
Ikeda Y., J. Fujii, M.E. Anderson, N. Taniguchi and A. Meister. 1995. Involvement of Ser-451 and Ser-452 in the catalysis of human gamma-glutamyl transpeptidase. J. Biol. Chem. 270: 22223-22228 .

Ikeda Y. and N. Taniguchi. 2005. Gene expression of gamma-glutamyltranspeptidase. Methods. Enzymol. 401: 408-425.

Marshall B.J. and J.R. Warren. 1984. Unidentified curved bacilli in the stomach of patients with gastritis and peptic ulceration. Lancet 1: 1311-1315.

McGovern K.J., T.G. Blanchard, J.A. Gutierrez, S.J. Czinn, S. Krakowka and P. Youngman. 2001. Gamma-glutamyltransferase is a Helicobacter pylori virulence factor but is not essential for colonization. Infect. Immun. 69: 4168-4173.

Sharma P. and N. Vakil. 2003. Review article: Helicobacter pylori and reflux disease. Aliment. Pharmacol. Ther. 17: 297-305.

Shibayama K., K. Kamachi, N. Nagata, T. Yagi, T. Nada, Y. Doi, N. Shibata, K. Yokoyama, K. Yamane, H. Kato, Y. Iinuma and Y. Arakawa. 2003. A novel apoptosis-inducing protein from Helicobacter pylori. Mol. Microbiol. 47: 443-451.

Su W., W. Shen, B. Li, Y. Wu, G. Gao and W. Wang. 2009. A novel way to purify recombinant baculoviruses by using bacmid. Bioscience report 29: 71-75 